\title{
Fellow Column: Comparing Transcutaneous Bilirubin Levels with Serum Bilirubin Levels to Screen for Neonatal Jaundice
}

Kaivan Dadachanji, DO

Newborn babies are frequently screened for jaundice to avoid kernicterus. The gold standard for checking for jaundice is the use of serum bilirubin levels and plotting the levels on an established nomogram (Bhutani Nomogram). (1, 2) However, unnecessary serum lab testing can be costly. Many clinics utilize a point of care transcutaneous bilirubin (TCB) to screen for jaundice. (3) Often clinics utilize a "75th percentile rule": if the TCB level is greater than $75 \%$ ile for age, total serum bilirubin (TSB) should be obtained. (4)

However, these TCB nomograms are based on old data; that do not account for updated devices. $(2,5)$

The TCB nomogram our clinic utilizes is based on data from Patras, Greece, using BiliChek Device between September 2005 and December 2007, which only included healthy, full-term infants with no NICU stay and minimal diversity in ethnic/ racial backgrounds. $(6,7)$ All patients were less than 120 hours old. 14,864 measurements from 2,818 patients.

\section{Materials and Methods:}

Retrospective data were obtained from August 2018 to January 2021 of patients in our Riverside University Health Systems Main General Pediatrics clinic. Pts must have TCB and TSB within 4 hours of each other. Our TCB data was obtained from 2 BiliChek ${ }^{\circledR}$ devices (manufactured by Philips).
"Newborn babies are frequently screened for jaundice to avoid kernicterus. The gold standard for checking for jaundice is the use of serum bilirubin levels and plotting the levels on an established nomogram (Bhutani Nomogram). (1, 2) However, unnecessary serum lab testing can be costly."

\section{Results:}

TCB, on average, is about 0.43 higher than TSB, p-value 0.0008 . $\mathrm{N}=754$. We found the TCB is more accurate for babies born $>92$ hours of life ( $p$-value 0.003 ). There is no change in variation between those born less than 92 hours of life and those greater than 92 hours of life. (Standard deviation: 1.67 vs 1.85, respectively; $F$ Value 1.22). No correlation between Hispanic and Non-Hispanic patients was found.

\section{Discussion:}

Further research should be obtained to determine how point-ofcare transcutaneous bilirubin devices can further minimize se-

TCB vs TSB

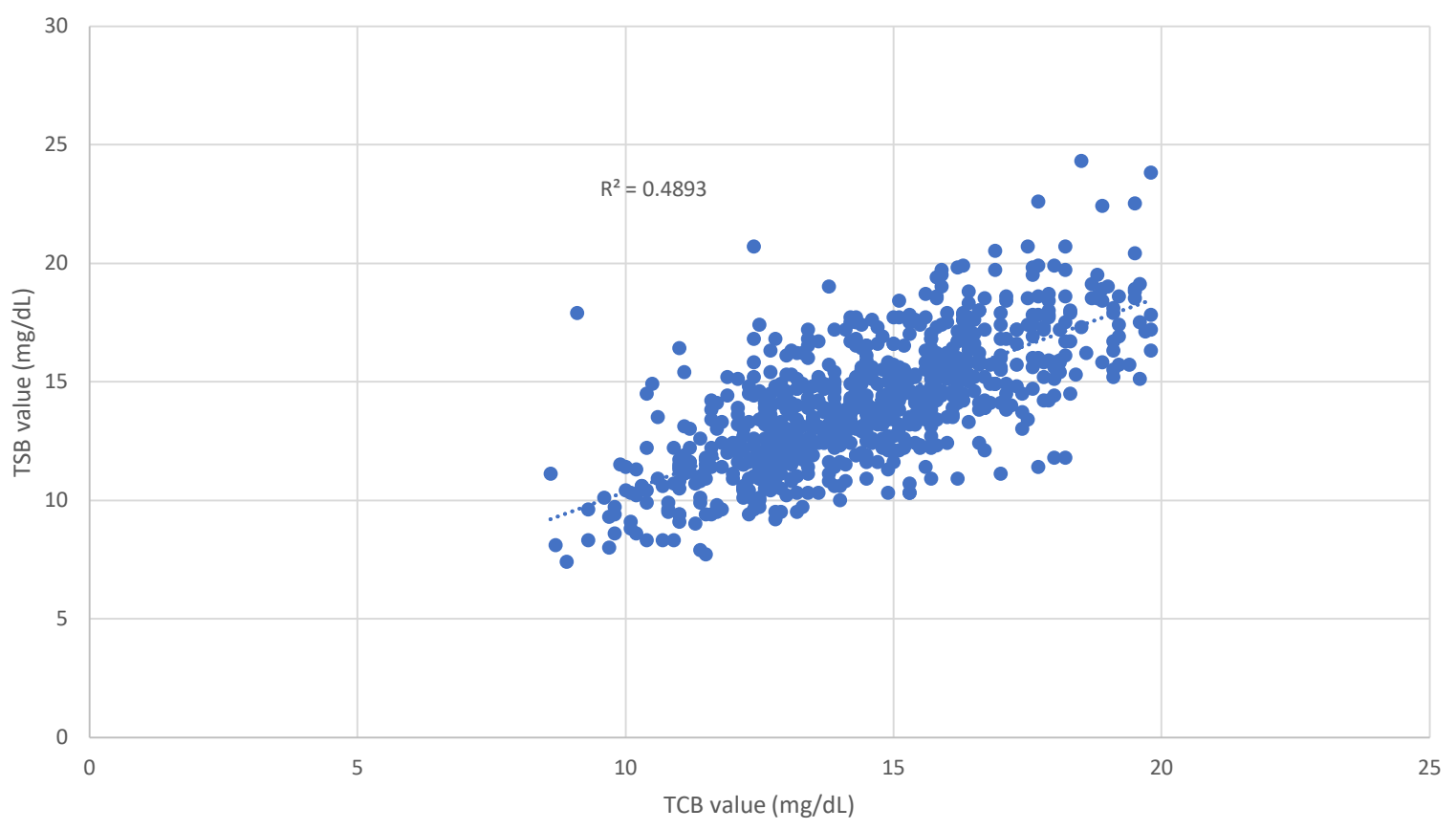


rum bilirubin testing. This may be achieved by creating new algorithms/ protocols that determine which transcutaneous bilirubin values. Our clinic is considering a quality improvement project to decrease total serum bilirubin blood draws safely. (4)

\section{References:}

1. Bhutani VK, Johnson L, Sivieri EM. Predictive ability of a predischarge hour-specific serum bilirubin for subsequent significant hyperbilirubinemia in healthy term and near-term newborns. Pediatrics. 1999;103(1):6-14. Epub 1999/01/26. doi: 10.1542/peds.103.1.6. PubMed PMID: 9917432.

2. Varvarigou A, Fouzas S, Skylogianni E, Mantagou L, Bougioukou D, Mantagos S. Transcutaneous bilirubin nomogram for prediction of significant neonatal hyperbilirubinemia. Pediatrics. 2009;124(4):1052-9. Epub 2009/09/30. doi: 10.1542/peds.2008-2322. PubMed PMID: 19786443.

3. Rizvi MR, Alaskar FM, Albaradie RS, Rizvi NF, Al-Abdulwahab K. A Novel Non-invasive Technique of Measuring Bilirubin Levels Using BiliCapture. Oman Med J. 2019;34(1):26-33. Epub 2019/01/24. doi: 10.5001/omj.2019.05. PubMed PMID: 30671181; PubMed Central PMCID: PMCPMC6330178.

4. Suresh GK, Clark RE. Cost-effectiveness of strategies that are intended to prevent kernicterus in newborn infants. Pediatrics. 2004;114(4):917-24. Epub 2004/10/07. doi: 10.1542/ peds.2004-0899. PubMed PMID: 15466085.

5. Fouzas S, Mantagou L, Skylogianni E, Mantagos S, Varvarigou A. Transcutaneous bilirubin levels for the first 120 postnatal hours in healthy neonates. Pediatrics. 2010;125(1):e52-7. Epub 2009/12/17. doi: 10.1542/ peds.2009-0403. PubMed PMID: 20008429.

6. Nagar G, Vandermeer B, Campbell S, Kumar M. Reliability of transcutaneous bilirubin devices in preterm infants: a systematic review. Pediatrics. 2013;132(5):871-81. Epub 2013/10/16. doi: 10.1542/peds.2013-1713. PubMed PMID: 24127472.

7. Zozaya $C$, Castro A. Transcutaneous bilirubin to guide phototherapy: authors' reply. J Perinat Med. 2019;47(6):682. Epub 2019/07/13. doi: 10.1515/jpm-2019-0220. PubMed PMID: 31299008.

Acknowledgments: Dr. Gabrielle Balan; Director of QI and Advocac, LLU/ RUHS Primary Care Tract. QI Project Mentor and Advisor. Dr. My Van Nguyen. Attending Faculty Mentor

Disclosure: The authors identify no conflict of interest

Funding Source: None

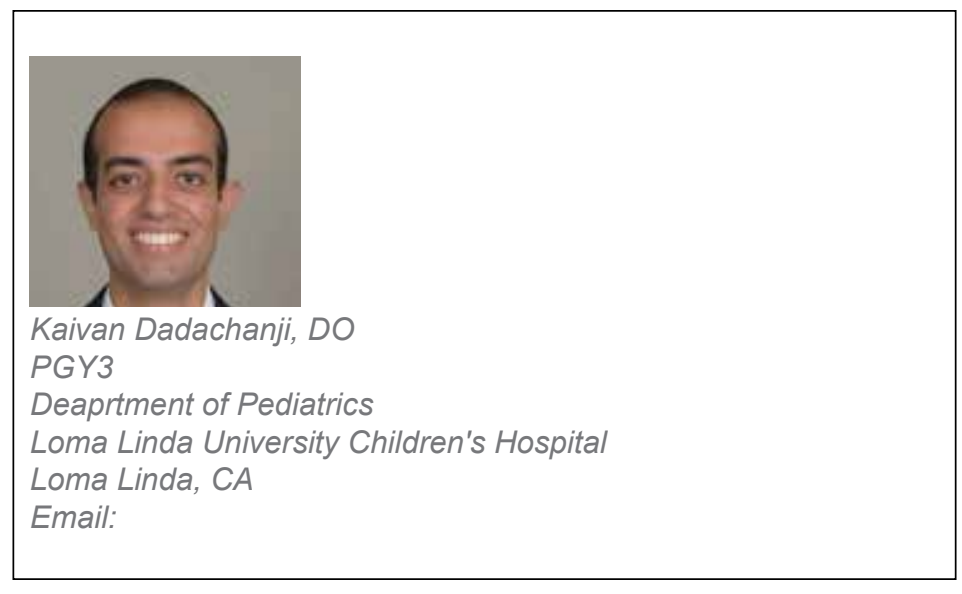

Fellow's Column is published monthly.

- Submission guidelines for "Fellow's Column":

- 2000 word limit not including references or title page. Exceptions will be made on a case by case basis

- $\quad$ QI/QA work, case studies, or a poster from a scientific meeting may be submitted..

- Submission should be from a resident, fellow, or NNP in training.

- Topics may include Perinatology, Neonatology, and Younger Pediatric patients.

- No more than 20 references.

- $\quad$ Please send your submissions to:

Elba Fayard, MD

Interim Fellowship Column Editor LomaLindaPublishingCompany@gmail.com
New subscribers are always welcome! NEOMATOLOGY

To sign up for a free monthly subscription, just click on this box to go directly to our subscription page 